\title{
THE CONNECTION MATRIX IN MORSE-SMALE FLOWS
}

\author{
JAMES F. REINECK
}

\begin{abstract}
In a Morse-Smale flow with no periodic orbits, it is shown that the connection matrix is unique. In the case of periodic orbits, nonuniqueness can occur. We show that on 2-manifolds, with some technical assumptions, given a connection matrix for the flow, one can replace the periodic orbits with doublyconnected rest points and obtain a new flow with no periodic orbits having the given connection matrix.
\end{abstract}

\section{INTRODUCTION}

The connection matrix is a collection of homomorphisms between the homologies of the Conley indices of the sets in a Morse decomposition of an isolated invariant set. It has been applied to several problems, including travelling wave solutions to reaction-diffusion equations $[9,12]$, and the study of ecological models [10]. The existence of connection matrices, and the fact that the connection matrix is not unique, were shown by Franzosa, who also proved the two continuation theorems stated subsequently. The idea of the continuation theorems is straightforward, but extremely important. It motivates the theorems, examples, and conjectures in this paper.

The purpose of this paper is to discuss the behavior of the connection matrix in Morse-Smale flows, i.e., flows where the Morse decomposition consists of hyperbolic rest points and periodic orbits, plus transversality. If there are no periodic orbits, then the connection matrix is unique. In the nontransverse case, uniqueness may fail, and the failure of uniqueness is a consequence of the continuation theorems. In the case of periodic orbits, nonuniqueness can occur in the transverse case, and this is also a consequence of the continuation theorem. We prove a theorem stating that on 2-manifolds, and with some (two-dimensional) technical assumptions, the only nonuniqueness which occurs is that forced by the continuation theorem.

In this section, we outline the theory of the connection matrix that will be used in the paper. In $\S 2$, we discuss the case where the Morse decomposition

Received by the editors November 9, 1987 and, in revised form, December 12, 1988.

1980 Mathematics Subject Classification (1985 Revision). Primary 58F12; Secondary 58F09, $34 \mathrm{C} 40$.

Key words and phrases. Conley index, connection matrix, continuation, Morse-Smale flow.

Part of the research was done while the author was at Northwestern University and supported by the National Science Foundation under grant no. DMS-852-1129. 
consists of hyperbolic rest points, proving the uniqueness theorem in the transverse case, and we also discuss the nontransverse case. In $\S 3$, we discuss the case of periodic orbits and prove the continuation theorem on 2-manifolds.

We first discuss some basic definitions from the Conley index theory. For a more complete discussion, see [1,2, 5, and 13].

Let $X$ be a topological space. A flow on $X$ is a continuous map $X \times \mathbb{R} \rightarrow X$, $(x, t) \rightarrow x \cdot t$ satisfying $x \cdot 0=x$ and $(x \cdot s) \cdot t=x \cdot(s+t)$. If $A \subset X$ and $J \subset \mathbb{R}$, we let $A \cdot J=\{x \cdot t \mid x \in A$ and $t \in J\}$. A set $S \subset X$ is called invariant if $S \cdot \mathbb{R}=S$. An invariant set $S$ is called an isolated invariant set if there is a compact neighborhood $N$ of $S$ such that $S$ is the maximal invariant set in $N$. Such an $N$ is called an isolating neighborhood for $S$. In Figure 1.1, the dashed rectangle is an isolating neighborhood for $S$ that consists of the two rest points and the connecting orbit. For $U \subset X$, we define $\omega(U)$, the $\omega$-limit set of $U$, to be the maximal invariant set contained in $\operatorname{cl}(U \cdot[0, \infty))$, and $\omega^{*}(U)$, the $\omega^{*}$-limit set of $U$, to be the maximal invariant set contained in $\operatorname{cl}(U \cdot(-\infty, 0])$. Let $S$ be an isolated invariant set.

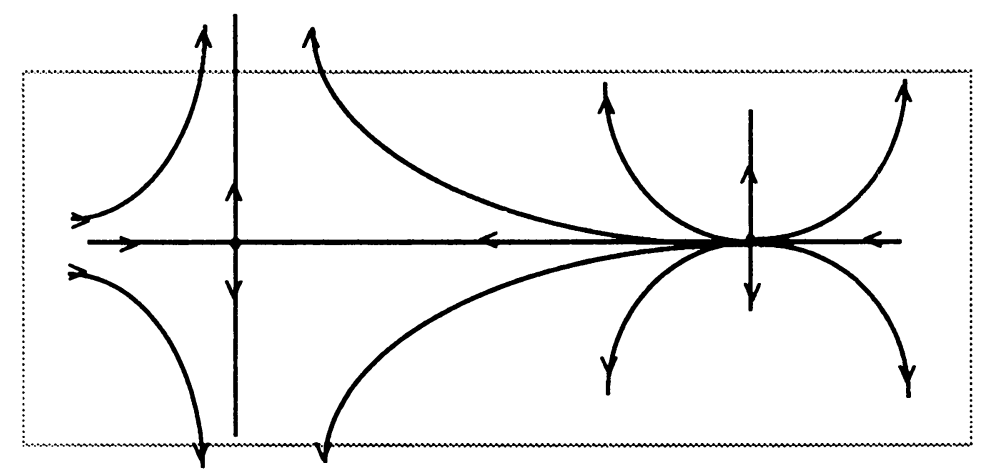

FigURE 1.1

Definition 1.2. $A \subset S$ is called an attractor if there is an $S$-neighborhood $U$ of $A$ such that $\omega(U)=A$. Similarly, a repeller in $S$ is a set that is the $\omega^{*}$-limit set of an $S$-neighborhood of itself.

Given an attractor $A \subset S$, define $A^{*}=\{x \in S \mid \omega(x) \cap A=\varnothing\}$. Then $A^{*}$ is a repeller, called the repeller dual to $A$, and the pair $\left(A, A^{*}\right)$ is called an attractor-repeller pair. The set of connecting orbits is

$$
C\left(A^{*}, A\right)=\left\{x \in S \mid \omega(x) \subset A \text { and } \omega^{*}(x) \subset A^{*}\right\} .
$$

It is not hard to see that $S=A \cup A^{*} \cup C\left(A^{*}, A\right)$. In Figure 1.1, the left rest point is an attractor, the right rest point its dual repeller.

Now we want to decompose $S$ into even smaller invariant sets. A finite set $P$ is partially ordered if there is a relation $<$ on $P$ satisfying

(i) for no $\pi \in P$ is $\pi<\pi$.

(ii) $\pi<\pi^{\prime}$ and $\pi^{\prime}<\pi^{\prime \prime}$ imply $\pi<\pi^{\prime \prime}$. 
We call $<$ a partial order on $P . I \subset P$ is an interval if $\pi, \pi^{\prime \prime} \in I, \pi^{\prime} \in P$, $\pi<\pi^{\prime}<\pi^{\prime \prime}$ implies $\pi^{\prime} \in P$.

Definition 1.3. Let $S$ be an isolated invariant set and $P$ a finite, partially ordered set. A Morse decomposition of $S$ is a collection $\left\{M_{\pi} \mid \pi \in P\right\}$ where each $M_{\pi}$ is an isolated invariant subset of $S$ and for all $x \in S \backslash \bigcup_{\pi \in P} M_{\pi}$ there is a $\pi<\pi^{\prime}$ in $P$ with $\omega(x) \subset \pi$ and $\omega^{*}(x) \subset \pi^{\prime}$. For a simple example of a Morse decomposition, consider the gradient flow for a Morse function on a compact manifold. In the general situation, the $M_{\pi}$ need not be rest points, but could be more complicated.

We now want to define an index for an isolated invariant set $S$. In a gradient flow, the Morse index of a critical point is the number of positive eigenvalues of the Hessian at the critical point. Conley generalized the index so that it is defined for an arbitrary isolated invariant set. The index is no longer a number, but takes the form of the homotopy type of a pointed topological space. If $Z \subset Y \subset X$, we say $Z$ is positively invariant relative to $Y$ if $x \in Z$, $x \cdot[0, t] \subset Y$ implies $x \cdot[0, t] \subset Z$.

Definition 1.4. A compact pair $\left(N_{1}, N_{0}\right)$ is called an index pair for $S$ if:

(i) $N_{1}$ is a neighborhood of $S$, and $\operatorname{cl}\left(N_{1} \backslash N_{0}\right)$ is an isolating neighborhood for $S$.

(ii) $N_{0}$ is positively invariant relative to $N_{1}$.

(iii) If $x \in N_{1}, x \cdot[0, \infty) \not \subset N_{1}$, then there is a $T \geq 0$ such that $x \cdot[0, T] \subset$ $N_{1}$ and $x \cdot T \in N_{0}$.

Property (iii) says that $N_{0}$ is the "exit set" of $N_{1}$, i.e., all points exiting $N_{1}$ do so via $N_{0}$. Index pairs exist. Their usefulness is shown by

Theorem 1.5 (Conley). If $\left(N_{1}, N_{0}\right)$ and $\left(N_{1}^{\prime}, N_{0}^{\prime}\right)$ are two index pairs for $S$, then there is a distinguished homotopy equivalence between the pointed spaces $N_{1} / N_{0}$ and $N_{1}^{\prime} / N_{0}^{\prime}$. The homotopy type of $N_{1} / N_{0}$ is called the Conley index of $S$ and is denoted $h(S)$.

For the rest points in Figure 1.1, we can take $N_{1}$ to be a small square around the rest point and $N_{0}$ to be the points on the boundary of $N_{1}$ that exit immediately in forward time. For the left rest point, the resulting quotient space has the homotopy type of a 1 -sphere, which we denote $\Sigma^{1}$, and for the right rest point, we get a 2 -sphere, $\Sigma^{2}$. The index of the entire isolated invariant set $S$ is computed by using the dashed rectangle as $N_{1}$ and the exiting boundary points as $N_{0}$. The index is the homotopy type of a disk, which is the same as a point. In general, if $S$ is a hyperbolic rest point, then $h(S)=\Sigma^{k}$, the pointed $k$-sphere where $k$ is the dimension of the unstable manifold of the point.

If $N \subset X$ is an isolated invariant set and we deform the flow in a continuous way such that $N$ remains an isolating neighborhood during the deformation, then the set $S_{0}$ isolated by $N$ before the deformation and the set $S_{1}$ that is isolated by $N$ after the deformation will have the same index. Indeed, there 
is a distinguished homotopy equivalence that depends on the deformation. We say $S_{0}$ and $S_{1}$ are related by elementary continuation. $S_{0}$ and $S_{1}$ are related by continuation if there is a sequence of elementary continuations beginning at $S_{0}$ and ending at $S_{1}$. Of course, $S_{0}$ and $S_{1}$ have the same index if they are related by continuation.

Let $\left\{M_{\pi} \mid \pi \in P\right\}$, a Morse decomposition of $S, I \subset P$ is interval. Define

$$
M(I)=\left(\bigcup_{\pi \in I} M_{\pi}\right) \cup\left(\bigcup_{\pi, \pi^{\prime} \in I} C\left(M_{\pi}, M_{\pi}^{\prime}\right)\right)
$$

$M(I)$ is an isolated invariant set. Franzosa [5] has shown that it is possible to construct a filtration of a neighborhood of $S$ so that one has index pairs for each $M(I)$ by choosing appropriate sets in the filtration. We call such a filtration an index filtration.

Notation. Let $\left\{M_{\pi} \mid \pi \in P\right\}$ be a Morse decomposition of an isolated invariant set $S$ with $P$ a partially ordered set. Choose an index filtration. Let $I \subset P$ be an interval, and let $\mathbb{F}$ be a field. Then

$$
\begin{aligned}
& C\left(M_{\pi}, M_{\pi^{\prime}}\right)=\left\{x \mid \omega^{*}(x) \subset M_{\pi}, \omega(x) \subset M_{\pi^{\prime}}\right\}, \\
& H(\pi)=H_{*}\left(h\left(M_{\pi}\right) ; \mathbb{F}\right) \quad \text { (singular homology), } \\
& H(I)=H_{*}(h(M(I)) ; \mathbb{F}) \quad \text { (singular homology). }
\end{aligned}
$$

The flow defines a partial order $<_{F}$ on $P$, namely, $\pi<_{F} \pi^{\prime}$ if $C\left(M_{\pi}, M_{\pi^{\prime}}\right) \neq$ $\varnothing$, and make the relation transitive. A partial order on $P$ that contains $<_{F}$ (as a subset of $P \times P$ ) is called an admissible ordering on $P$.

We briefly outline the portions of the connection matrix theory (due to Franzosa) that we shall use. Details can be found in, [4 and 6]. If $\left(A, A^{*}\right)$ is an attractor-repeller pair in an isolated invariant set $S$, we can find a compact triple of spaces $\left(N_{2}, N_{1}, N_{0}\right)$ such that $\left(N_{2}, N_{0}\right)$ is an index pair for $S,\left(N_{1}, N_{0}\right)$ is an index pair for $A$, and $\left(N_{2}, N_{1}\right)$ is an index pair for $A^{*}$. We consider $N_{2} / N_{1}$ as a pointed space with the equivalence class of $N_{1}$ as the distinguished point, and similarly for the other two pairs. There is a long exact homology sequence of pointed spaces

$$
\cdots \rightarrow H_{q}\left(N_{1} / N_{0}\right) \rightarrow H_{q}\left(N_{2} / N_{0}\right) \rightarrow H_{q}\left(N_{2} / N_{1}\right) \stackrel{\partial}{\rightarrow} H_{q-1}\left(N_{1} / N_{0}\right) \rightarrow \cdots .
$$

Since this is essentially independent of the triple, we write

$$
\cdots \rightarrow H_{q}(A) \stackrel{i}{\rightarrow} H_{q}(S) \stackrel{p}{\rightarrow} H_{q}\left(A^{*}\right) \stackrel{\partial}{\rightarrow} H_{q-1}(A) \rightarrow \cdots
$$

where $H_{q}(S)$ denotes $H_{q}(h(S))$, etc. We call $\partial$ the flow-defined boundary map. Exactness implies that if $H(S)=0$, then $\partial$ is an isomorphism. If $C\left(A^{*}, A\right)=\varnothing$, then $H(S)=H(A) \oplus H\left(A^{*}\right)$, and it follows that $\partial$ is identically zero. So we have the following lemma. 
Lemma 1.7. If $\partial \neq 0$, then $C\left(A^{*}, A\right) \neq \varnothing$.

Given a Morse decomposition $\left\{M_{\pi} \mid \pi \in P\right\}$, if $\pi$ and $\pi^{\prime}$ are adjacent in an admissible order with $\pi<\pi^{\prime}$, then $\left(M(\pi), M\left(\pi^{\prime}\right)\right)$ forms an attractor-repeller pair in $M\left(\pi, \pi^{\prime}\right)$. Similarly, if $(I, J)$ is an adjacent pair of intervals (i.e., $I \cup J$ is an interval and no element of $J$ lies below one in $I)$, then $(M(I), M(J))$ forms an attractor-repeller pair in $M(I \cup J)$. In both cases we get sequences as in (1.6) and flow-defined boundary maps $\partial\left(\pi^{\prime}, \pi\right)$ and $\partial(J, I)$. The connection matrix puts restrictions on the maps $\partial\left(\pi^{\prime}, \pi\right)$, and aliows us to compute unknown maps from known ones. For an interval $I$ in $P$, we define

$$
C \Delta(I)=\bigoplus_{\pi \in I} H(\pi) .
$$

A map $\Delta(I): C \Delta(I) \rightarrow C \Delta(I)$ can be thought of as a matrix

$$
\left[\Delta\left(\pi, \pi^{\prime}\right): H(\pi) \rightarrow H\left(\pi^{\prime}\right) \mid \pi^{\prime}, \pi \in P\right]
$$

indexed by pairs of elements of $I . \Delta(P)$ is called upper triangular if $\Delta\left(\pi^{\prime}, \pi\right)=$ 0 if $\pi \nless \pi^{\prime} . \Delta(P)$ is called a boundary map if each $\Delta\left(\pi^{\prime}, \pi\right)$ has degree -1 and $(\Delta(P))^{2}=0$. It is not difficult to show that if $\Delta(P)$ is an upper triangular boundary map, so is the restriction $\Delta(I)$ to any interval $I$. Thus, $(C \Delta(I), \Delta(I))$ is a chain complex. Let $H \Delta(I)$ denote its homology. There are obvious inclusions and projections that make

$$
0 \rightarrow C \Delta(I) \rightarrow C \Delta(I \cup J) \rightarrow C \Delta(J) \rightarrow 0
$$

a short exact sequence of chain complexes, so there is an exact homology sequence

$$
\cdots \rightarrow H \Delta(I) \stackrel{i}{\rightarrow} H \Delta(I \cup J) \stackrel{p}{\rightarrow} H \Delta(J) \stackrel{\partial}{\rightarrow} H \Delta(I) \rightarrow \cdots
$$

An upper triangular boundary map $\Delta(P)$ is called a connection matrix if for each interval $I$ there is a homomorphism $\Phi(I): H \Delta(I) \rightarrow H(I)$, and the $\Phi(I)$ satisfy the following conditions:

(a) for $\pi \in P, \Phi(\pi): H \Delta(\pi)=H(\pi) \rightarrow H(\pi)$ is the identity;

(b) for each adjacent pair of intervals $(I, J)$, the following diagram commutes:

$$
\begin{aligned}
& \cdots \rightarrow H \Delta(I) \stackrel{i}{\longrightarrow} H \Delta(I \cup J) \stackrel{p}{\longrightarrow} H \Delta(J) \stackrel{\partial}{\longrightarrow} H \Delta(I) \rightarrow \cdots \\
& \Phi(I) \downarrow \quad \Phi(I \cup J) \downarrow \quad \Phi(J) \downarrow \quad \Phi(I) \downarrow \\
& \cdots \rightarrow H(I) \stackrel{i}{\longrightarrow} H(I \cup J) \stackrel{p}{\longrightarrow} H(J) \stackrel{\partial}{\longrightarrow} H(I) \rightarrow \cdots
\end{aligned}
$$

By induction and the five lemma, each $\Phi(I)$ is an isomorphism. Condition (a) says that if $I=\pi^{\prime}$ and $J=\pi$, then $\Delta\left(\pi, \pi^{\prime}\right)=\partial\left(\pi, \pi^{\prime}\right)$, i.e., the connection matrix entries between adjacent Morse sets are the flow-defined boundary maps. If $<$ is an admissible order on $P$, we let $C M(<)$ denote the set of connection matrices with the order $<$. The following is easy to show. 
Lemma 1.9. If $<$ is an admissible order, then $C M\left(<_{F}\right) \subset C M(<)$.

In this paper, we shall analyze the maps that are not flow defined, i.e., maps between Morse sets that are not adjacent in the flow-defined partial order.

We will need some facts from the continuation theory of the connection matrix. Suppose we have a one-parameter family of flows, parameterized by the interval $[0,1]$. Assume that for each $\lambda \in[0,1]$, we have an isolated invariant set $S^{\lambda}$, and a Morse decomposition $\left\{M_{\pi}^{\lambda} \mid \pi \in\left(P,<_{\lambda}\right)\right\}$ of $S^{\lambda}$, where $<_{\lambda}$ denotes the flow-defined order at parameter value $\lambda$, and assume that the $S^{\lambda}$ and $M_{\pi}^{\lambda}$ are all related by continuation. We do not assume that the flowdefined order is constant. For $I$ an interval in $P$ and $\lambda \in[0,1]$, we define $H_{\lambda}(I)=H_{*}\left(h\left(M_{\lambda}(I)\right) ; \mathbb{F}\right)$. If $<_{0}$ is an admissible order for parameter values between 0 and $\lambda$, then there are flow-defined isomorphisms

$$
\theta(I)^{-1}: H_{0}(I) \rightarrow H_{\lambda}(I)
$$

for each interval $I$. Given a connection matrix for the flow at parameter value $\lambda$, we get a connection matrix for the flow at parameter value 0 via

$$
\Delta_{0}\left(\pi, \pi^{\prime}\right)=\theta\left(\pi^{\prime}\right)^{-1} \Delta_{\lambda}\left(\pi, \pi^{\prime}\right) \theta(\pi) .
$$

This gives a map $\Theta: C M_{\lambda}\left(<_{\lambda}\right) \rightarrow C M_{0}\left(<_{0}\right)$. This map is injective if $<_{0}$ remains an admissible order between 0 and $\lambda$, which is always true if $\lambda$ is small enough.

Theorem 1.10 [7]. If $<_{0}$ remains an admissible order between 0 and $\lambda$, then $\Theta: C M_{\lambda}\left(<_{\lambda}\right) \rightarrow C M_{0}\left(<_{0}\right)$ is injective, i.e., $C M_{\lambda}\left(<_{\lambda}\right) \subset C M_{0}\left(<_{0}\right)$. If $\lambda$ is small enough, the inclusion holds.

If $<_{0}$ is the flow-defined order between 0 and $\lambda$, we can reverse the argument and obtain the following:

Theorem 1.11 [7]. If $<_{0}$ remains the flow-defined order between 0 and $\lambda$, then $\Theta: C M_{\lambda}\left(<_{\lambda}\right) \rightarrow C M_{0}\left(<_{0}\right)$ is a bijection, i.e., $C M_{\lambda}\left(<_{\lambda}\right)=C M_{0}\left(<_{0}\right)$.

As we will illustrate in the next section, the flow-defined order need not continue to nearby $\lambda$.

We conclude with two facts that we will need. The first is a result of McCord [8].

Theorem 1.12. Suppose $\mathbb{F}=\mathbb{Z}_{2}, A$ and $A^{*}$ are hyperbolic rest points with Conley indices $\Sigma^{k}$ and $\Sigma^{k+1}$, respectively, and the stable and unstable manifolds intersect transversally. Then the flow-defined map $\partial$ counts the number of connecting orbits from $A^{*}$ to $A(\bmod 2)$.

A similar statement holds for more general coefficients, provided one considers orientations. The second fact is a general statement about Morse decompositions. 
Lemma 1.13. Suppose $\left\{M_{\pi} \mid \pi \in P\right\}$ is a Morse decomposition of an isolated invariant set $S,\left\{x_{n}\right\}$ is a sequence in $S, \omega\left(x_{n}\right) \subset M_{\pi}$ for each $n$ and $x_{n} \rightarrow x$. Then $\omega(x) \subset M_{\pi^{\prime}}$ for some $\pi^{\prime}$, and $\pi \leq \pi^{\prime}$ in the flow-defined order. Similarly, if $\omega^{*}\left(x_{n}\right) \subset M_{\rho}$ for each $n$, then $\omega^{*}(x) \subset M_{\rho^{\prime}}$, and $\rho^{\prime} \leq \rho$.

Proof. We prove the assertion for $\omega(x)$. The other statement is similar. That $\omega(x) \subset M_{\pi^{\prime}}$ for some $\pi^{\prime}$ is shown in [13], so it remains to show that $\pi \leq \pi^{\prime}$. Suppose this is not the case. Let $I=\left\{\alpha \in P \mid \alpha \leq \pi^{\prime}\right\}$. Then $I$ is an interval, $M(I)$ is an attractor, and $M(I) \cap M_{\pi}=\varnothing$. Let $U$ be an attractor neighborhood of $M(I)$, i.e., $U$ is a neighborhood of $M(I)$ and $\omega(U)=M(I)$. For $n$ large enough, the forward orbit of $x_{n}$ enters $U$, so the same must be true for $x$. This implies that $\omega(x) \subset M(I)$, contradicting the fact that $\omega(x) \subset M_{\pi^{\prime}}$.

\section{THE CASE OF HYPERBOLIC REST POINTS}

Throughout this section, we assume that $\left\{M_{\pi} \mid \pi \in P\right\}$ is a Morse decomposition of an isolated invariant set $S$ contained in a local flow in a smooth manifold, and that each $M_{\pi}$ is a hyperbolic rest point. Since each Morse set is a hyperbolic rest point, the index of each set is a pointed sphere $\Sigma^{k}$, where $k$ is the dimension of the unstable manifold of the point. Let $W^{u}(\pi)$ and $W^{s}(\pi)$ denote the stable and unstable manifold of $M_{\pi}$. In the case of transversality we have the following:

Theorem 2.1. Suppose $W^{s}(\pi)$ and $W^{u}\left(\pi^{\prime}\right)$ intersect transversally for all $\pi$, $\pi^{\prime} \in P$. Then the connection matrix $\Delta$ (in the flow-defined order) is unique.

Proof. Suppose $\pi, \pi^{\prime} \in P, \pi^{\prime}<\pi$, where $<$ denotes the flow-defined order. If $\pi^{\prime}$ and $\pi$ are adjacent in $P$, then the map $\Delta\left(\pi, \pi^{\prime}\right)$ is defined by a sequence as in (1.6), so it is unique. We will show that $\Delta\left(\pi, \pi^{\prime}\right)=0$ if $\pi^{\prime}$ and $\pi$ are not adjacent.

Suppose $\pi^{\prime}<\pi$, but $\pi^{\prime}$ and $\pi$ are not adjacent. Then there is a $\pi^{\prime \prime} \in P$ with $\pi^{\prime}<\pi^{\prime \prime}<\pi$. Let $i, j$, and $k$ be such that $h(\pi)=\Sigma^{i}, h\left(\pi^{\prime \prime}\right)=\Sigma^{j}$, and $h(\pi)=\Sigma^{k}$. Since $\pi^{\prime}<\pi^{\prime \prime}$ and $W^{s}\left(\pi^{\prime}\right)$ intersects $W^{u}\left(\pi^{\prime \prime}\right)$ transversally, we have $i<j$, i.e., $i \leq j-1$. Similarly, $j \leq k-1$, so $i \leq k-2$. The map $\Delta\left(\pi, \pi^{\prime}\right)$ is a map of degree -1 from $H_{*}\left(\Sigma^{k}, \mathrm{pt} ; \mathbb{F}\right)$ to $H_{*}\left(\Sigma^{i}, \mathrm{pt} ; \mathbb{F}\right)$, where $i \leq k-2$. The only possible map is $\Delta\left(\pi, \pi^{\prime}\right)=0$. Thus, each map in $\Delta$ is either 0 or defined uniquely by the flow.

The following example shows that the transversality assumption is necessary in Theorem 2.1. The example is well known, but the nonuniqueness argument is different from that in [6].

Example 2.2. Consider the flow in Figure 2.3. $S$ consists of the three rest points plus the connecting orbits. We have $h(1)=\Sigma^{0}, h(1)=h(2)=\Sigma^{1}$. The flow-defined order is $1<2,2<3$, and $1<3$. The maps $\Delta(2,1)$ and $\Delta(3,2)$ are flow defined, but $\Delta(3,1)$ is not. It is not hard to see that $h(1,2)$ is $\overline{0}$, 


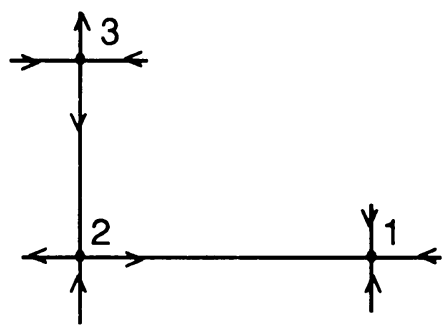

FIGURE 2.3

the pointed one-point space, so $H(1,2)=0$. Putting this into (1.6) gives that $\Delta(2,1)$ is an isomorphism. $H(2)$ and $H(3)$ are only nonzero in dimension one, so $\Delta(3,2)$ is 0 . So $\Delta$ has the following form:

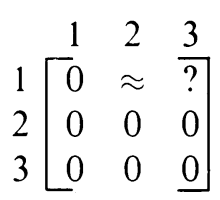

We will use Theorem 1.5 to show that $\Delta(3,1)$ can be 0 or an isomorphism.
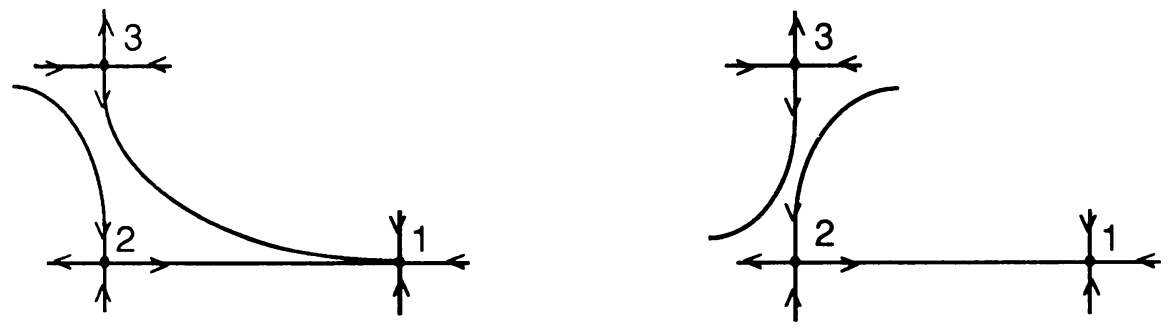

FIGURE 2.4

Consider Figure 2.4a. Such a flow can be realized by an arbitrarily small perturbation of the flow in Figure 2.3. In this case, the partial order is $1<2$ and $1<3$. The map $\Delta(3,1)$ is now flow defined, and it is easy to see that $h(1,3)=\overline{0}$, so $H(1,3)=0$ and $\Delta(3,1)$ is an isomorphism. On the other hand, if we perturb the flow as in Figure $2.4 \mathrm{~b}$, then $\Delta(3,1)=0$ since $1 \nless 3$. Since each of these connection matrices can be realized by arbitrarily small perturbations of the flow in Figure 2.3, Theorem 1.10 implies that both of these are connection matrices for the flow in Figure 2.3.

One may ask if the converse of Theorem 2.1 is true. The following example shows that the answer is no.

Example 2.5. For this example, we let $\mathbb{F}=\mathbb{Z}_{2}$. Consider the flow in Figure 2.6. This flow consists of two copies of the flow in Figure 2.3; the indices of the rest points, as well as the partial order, are as in that example. This time 
$\Delta(2,1)=0$. This can be seen by using an annulus to isolate $M(1,2)$. We have $h(M(1,2))=$ disjoint union of a circle and a point, and it follows that $H(1,2)=H(1) \oplus H(2)$, so by using (1.6), we have $\Delta(2,1)=0$. The total index $h(S)=\Sigma^{1}$, so $H(S) \neq H(1) \oplus H(2) \oplus H(3)$, and $\Delta(3,1) \neq 0$. Since $\mathbb{F}=\mathbb{Z}_{2}$, the only other possibility is $\Delta(3,1)=1$. Thus $\Delta$ is unique, but $W^{u}(3)$ and $W^{s}(2)$ do not intersect transversally.

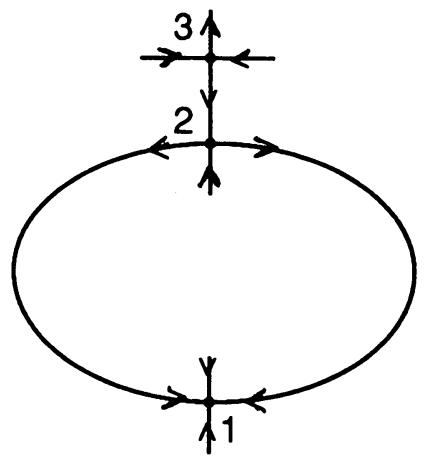

FIGURE 2.6

In this example, the fact that $\mathbb{F}=\mathbb{Z}^{2}$ was essential, but it is not difficult to construct an analogous example in $\mathbb{R}^{3}$ where $\Delta$ is unique in a field of any characteristic, but the intersections are not transverse. In this setting, $h(M(1))=\Sigma^{1}, h(M(2))=h(M(3))=\Sigma^{2}$, and $h(M(1,2))=\mathbb{R} P^{2}$.

We make two final remarks. First, notice that in the setting of Theorem 2.1 , the follow is structurally stable, so the partial order does not change under small perturbations, and Theorem 1.11 applies. Second, a possible theorem in the nontransverse case is the following. Given a flow with an isolated invariant set, a Morse decomposition consisting of hyperbolic rest points, a neighborhood $N$ of the flow in the compact-open (or $C^{1}$ ) topology, and a connection matrix $\Delta$, there is a flow in $N$ that has transverse intersections and that has $\Delta$ as its unique connection matrix. We have not attempted to prove this. However, such a theorem would indicate that all of the ambiguity in the connection matrix is forced by Theorem 1.10. If one can find a connection matrix $\Delta$ in every neighborhood of a given flow, then $\Delta$ must be a connection matrix for the given flow (as in Example 2.2).

\section{PERIODIC ORBITS}

Suppose $S$ is an isolated invariant set, the Morse decomposition consists of hyperbolic rest points and periodic orbits, and the stable and unstable manifolds intersect transversally, i.e., $S$ is in a Morse-Smale flow. This is the simplest 
generalization of the situation in Theorem 2.1. Throughout this section we use $\mathbb{F}=\mathbb{Z}_{2}$. The following example shows that the connection matrix is not unique.

Example 3.1. Consider the local flow in $\mathbb{R}^{2}$ which is generated by

$$
\dot{r}=r(r-1)(r-2), \quad \dot{\theta}=\varphi(r) \sin 2 \theta+(1-\varphi(r))
$$

where $\varphi \equiv 1$ on $[3 / 2,5 / 2]$ and $\varphi \equiv 0$ outside of $[5 / 4,3]$. The picture is Figure 3.3. Let $N=\{(r, \theta) \mid 1 / 2 \leq r \leq 5 / 2\}$. Then $N$ is an isolating neighborhood, and it isolates $S=\{(r, \theta) \mid 1 \leq r \leq 2\}$. Using $N$ and its outer boundary circle as an index pair, we see that $h(S)=\overline{0}$, the pointed one-point space. The periodic orbit and four rest points form a Morse decomposition of $S$, with flow-defined order $1<2,3,4,5 ; 2<4,5$; and $3<4,5$. Thus, $\{1,4\}$ and $\{1,5\}$ are not intervals, and the maps $\Delta(4,1)$ and $\Delta(5,1)$ are not flow defined. The index $h(1)$ is the disjoint union of a circle and a distinguished point, so $H_{q}(1)=\mathbb{Z}_{2}$ if $q=0$ or 1 . The other indices are spheres, $h(2)=h(3)=\Sigma^{1}, h(4)=h(5)=\Sigma^{2}$. If we consider the flow as a flow on the two-sphere, then $\infty$ is an attractor and the transversality condition is clearly satisfied. It is easy to compute the flow-defined boundary maps using index triples. The maps from the repellers to the saddles and from the saddles to $H_{0}(1)$ are all isomorphisms. The map from $H_{1}(1)$ to $H_{0}(1)$ is 0 by upper triangularity. Thus, the connection matrix looks like this:

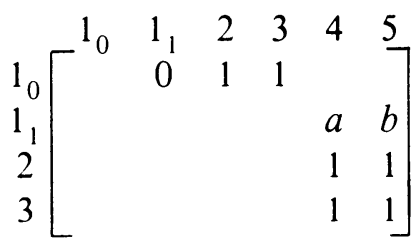

with the entries $a$ and $b$ not flow defined. $H(S)=0$, so the rank of $\Delta$ must equal the dimension of the kernel of $\Delta$. It follows that $a$ and $b$ are different, i.e., $a=1$ and $b=0$, or $a=0$ and $b=1$. For either choice of $a$ and $b$ it is not difficult to construct $\Phi$ 's to make diagram (1.8) commute for all adjacent pairs of intervals. Also, symmetry would suggest that both choices yield connection matrices. Thus uniqueness fails in the case of periodic orbits.

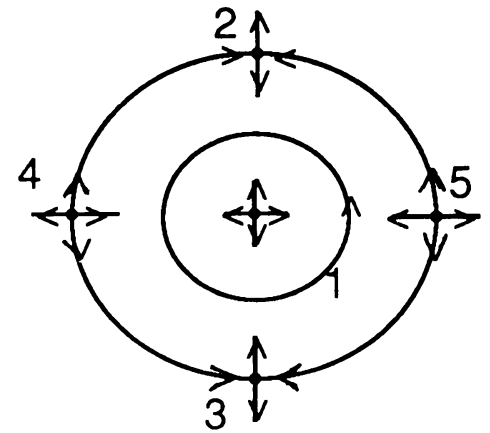

FIGURE 3.3 
To understand this phenomenon, consider the following system:

$$
\begin{gathered}
\dot{r}=r(r-1)(r-2), \\
\dot{\theta}=\varphi_{1}(r)+\varphi_{2}(r) \sin (\theta+\alpha)+\varphi_{3}(r) \sin 2 \theta,
\end{gathered}
$$

where $\varphi_{1}(r)+\varphi_{2}(r)+\varphi_{3}(r)=1, \varphi_{2}(r) \equiv 1$ near 1 and supported near 1 , $\varphi_{3}(r) \equiv 1$ near 2 and supported near 2 , and $\alpha \in[0,2 \pi)$. The picture is Figure 3.5. Here we have continued the periodic orbit to two doubly-connected rest points. Since the partial order continues, we have, if we consider the new rest points and connections as a single Morse set, the same two connection matrices as in (3.2). However, there is a finer Morse decomposition, namely, the six rest points. For all but two values of $\alpha$, there will be no saddle-saddle connections, so we will be in the case of Theorem 2.1. This flow with the finer decomposition will therefore have a unique connection matrix. This connection matrix will be a connection matrix for the flow (3.2). This is shown subsequently and follows intuitively from Theorem 1.10. By varying $\alpha$, we can have the newly created saddle connected to either 4 or 5 . If the new saddle is connected to 4 , then we have $a=1$ and $b=0$, and if the new saddle is connected to 5 , we have $a=0$ and $b=1$. Thus, the nonuniqueness is required by the two different continuations and Theorem 1.10.

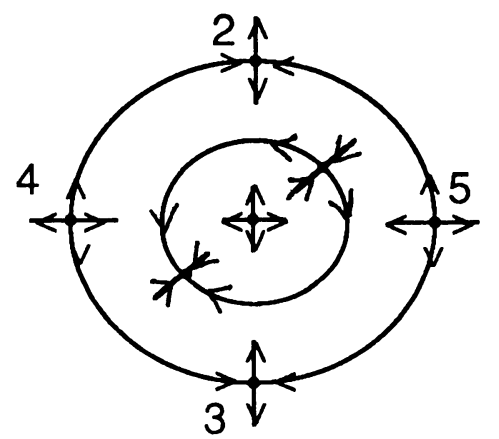

FIGURE 3.5

The question now arises as to whether all of the nonuniqueness in MorseSmale flows arises in this way. The following example shows that this is not the case.

Example 3.6. We consider the flow obtained by replacing the $\sin 2 \theta$ term in (3.2) with $\sin 3 \theta$. It is shown in Figure 3.7. $S$ is again the set $\{1 \leq r \leq 2\}$, and the rest points plus the periodic orbits form a Morse decomposition. All the maps are flow defined except $\Delta(5,1), \Delta(6,1)$, and $\Delta(7,1)$. The flow-defined 
maps are easily calculated, and we have the following connection matrix:

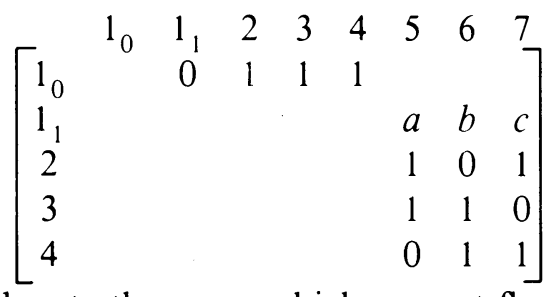

where $a, b$, and $c$ denote the maps which are not flow defined. Since $H(S)$ is 0 , the rank of $\Delta$ must be 4 . It follows that $a+b+c=1$. There are four possibilities, namely, that one of the entries is one or all of the entries are one. Again one can construct the $\Phi$ 's to make diagram (1.8) commute for all adjacent pairs of intervals, so there are four connection matrices. However, if we continue the periodic orbit to a doubly-connected saddle and attracting rest point, then the new saddle cannot be connected to all three of the repellers since there are only two orbits in the stable manifold of the saddle. Thus, the connection matrix with $a=b=c=1$ cannot be obtained by continuing the periodic orbits to two doubly-connected rest points as in Example 3.1. Clearly the problem here occurs because the saddle has only two orbits in the stable manifold, but the algebra depends only on the differences in the dimensions of the stable manifolds.

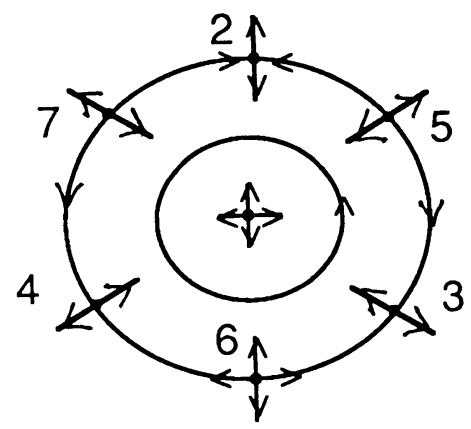

FIGURE 3.7

Except for the problem of Example 3.6, and one other (one-dimensional) assumption, all of the ambiguity in the connection matrix for Morse-Smale flows in dimension 2 is forced by Theorem 1.10 in the same way that the nonuniqueness occurred in Example 3.1. We begin the proof of this by establishing some terminology.

Definition 3.8. Let $\mathscr{U}=\left\{U_{1}, U_{2}, \ldots, U_{n}\right\}$ be a collection of neighborhoods of the periodic orbits $\gamma_{1}, \gamma_{2}, \ldots, \gamma_{k}$ in the Morse-Smale flow $\varphi$ such that $\gamma_{i} \subset U_{i}$ and $U_{i}$ is disjoint from every other set in the Morse decomposition. We call the flow $\varphi^{\prime}$ a $\mathscr{U}$-refinement of $\varphi$ if:

(a) $\varphi^{\prime}$ is Morse-Smale. 
(b) $\varphi^{\prime}$ agrees with $\varphi$ outside of $\bigcup U_{i}$.

(c) In each $U_{i}, \varphi^{\prime}$ has two rest points $\{p, q\}$ of index $k$ and $k+1$ where $k+1$ is the dimension of the unstable manifold of $\gamma_{i}$. There are exactly two orbits connecting the two rest points in $U_{i}$ and no other rest points or periodic orbits in $U_{i}$. Finally, the $W^{u}\left(\gamma_{i}\right)$ in $\varphi$ equals $W^{u}(p) \cup W^{u}(q)$ in $\varphi^{\prime}$ and $W^{s}\left(\gamma_{i}\right)$ in $\varphi$ equals $W^{s}(p) \cup W^{s}(q)$ in $\varphi^{\prime}$.

The idea of such a refinement is due to Franks [3].

Remark 3.9. Suppose $\varphi^{\prime}$ is a $\mathscr{U}$ refinement of $\varphi$, and $\left\{M_{\pi} \mid \pi \in P\right\}$ is a Morse decomposition of $S$ in $\varphi$. Then there is a Morse decomposition $\left\{M_{\pi}^{\prime} \mid \pi \in P\right\}$ of $S$ in $\varphi^{\prime}$ as follows. $M_{\pi}^{\prime}=M_{\pi}$ if $M_{\pi}$ is a rest point, and if $M_{\pi}$ is a periodic orbit $\gamma$, then $M_{\pi}^{\prime}$ is the two rest points and two connecting orbits that replaced $\gamma$. However, there is a finer Morse decomposition for $S$ in $\varphi^{\prime}$, namely, the rest points. Denote this Morse decomposition by $\left\{M_{\rho}^{\prime \prime} \mid \rho \in R\right\}$. Each periodic orbit in the collection $\left\{M_{\pi} \mid \pi \in P\right\}$ has Conley index $\Sigma^{k} \vee \Sigma^{k+1}$. (Actually, if $k=0$, then the index is the disjoint union of a circle containing the distinguished point and another point), so each periodic orbit corresponds to two rest points in the Morse decomposition $\left\{M_{\rho}^{\prime \prime} \mid \rho \in R\right\}$. Let $\Delta$ be a connection matrix for $S$ in $\varphi^{\prime}$ with the Morse decomposition $\left\{M_{\rho}^{\prime \prime} \mid \rho \in R\right\}$. Then $\Delta$ can be thought of as a map on the chain complex $C \Delta(P)$ which comes from the Morse decomposition $\left\{M_{\pi} \mid \pi \in P\right\}$. For such a $\Delta$ we have the following:

Theorem 3.10. If the $U_{i}$ in $\mathscr{U}$ are small enough, then $\Delta$ is a connection matrix for the Morse decomposition $\left\{M_{\pi} \mid \pi \in P\right\}$ of $S$.

Proof. We first show that $\Delta$ is a connection matrix for $S$ in $\varphi^{\prime}$ with the Morse decomposition $\left\{M_{\pi}^{\prime} \mid \pi \in P\right\}$. If $p$ and $q$ are two rest points that replaced a periodic orbit in the refined Morse decomposition, then $\Delta(q, p)=0$ by Theorem 1.12, so $\Delta$ is upper triangular on $P$. Thus, $\Delta$ is an upper triangular boundary map. If $I$ is an interval in $P$, then $I$ is an interval in $R$, and the maps in the lower row of diagram (1.8) are the same, so the $\Phi(I)$ that makes diagram (1.8) commute for $\left\{M_{\rho}^{\prime \prime} \mid \rho \in R\right\}$ also make the diagram commute for $\left\{M_{\pi}^{\prime} \mid \pi \in P\right\}$. It remains to show that $\Phi(\pi)=$ id for $\pi \in P$. If $M_{\pi}^{\prime}$ is a rest point, then $\Phi(\pi)=\Phi(\rho)=$ id. If $M_{\pi}^{\prime}$ is two rest points and two connecting orbits, then we have $\Phi(\pi)=\Phi(p, q)$ where $p$ and $q$ are the rest points. If $H_{k} \Delta(p, q)$ is nonzero, then it is one dimensional, so the only possible nonzero $\Phi$ is the identity in dimension $k$. Thus, $\Phi(\pi)=\Phi(p, q)=\mathrm{id}$, and $\Delta$ is a connection matrix for $\left\{M_{\pi}^{\prime} \mid \pi \in P\right\}$. If the $U_{i}$ in $\mathscr{U}$ are small enough, an index filtration for $\left\{M_{\pi}^{\prime} \mid \pi \in P\right\}$ in $\varphi^{\prime}$ will be an index filtration for $\left\{M_{\pi} \mid \pi \in P\right\}$ in $\varphi$, so the connection matrices for $\left\{M_{\pi}^{\prime} \mid \pi \in P\right\}$ are the same as those for $\left\{M_{\pi} \mid \pi \in P\right\}$ by Theorem 1.10. Thus, $\Delta$ is a connection matrix for $S$ with Morse decomposition $\left\{M_{\pi} \mid \pi \in P\right\}$ 
The interesting question is the converse of Theorem 3.10: Given a connection matrix $\Delta$ for a Morse-Smale flow, and a collection $\mathscr{U}$, can one find a $\mathscr{U}$ refinement $\varphi^{\prime}$ which has $\Delta$ as its unique connection matrix? Example 3.6 shows that the answer is no in general, but if we ignore the connection matrices that have a saddle connected to more than two attractors (or repellers) and add one other assumption (which is needed because the boundary of an annulus is not connected), then the answer for Morse-Smale flows on 2-manifolds is yes. For the rest of the paper, we will assume that the flow is on a 2-manifold.

Definition 3.11. Let $\gamma$ be a periodic orbit, and let $V$ be a tubular neighborhood of $\gamma$. Then $V$ is either an annulus, in which case we call $\gamma$ orientable, or $V$ is a Möbius band, in which case $\gamma$ is not orientable. We say an orientable $\gamma$ is in standard form if there are coordinates $(\theta, x)$ in $V=S^{1} \times[-1,1]$ such that the vector field has this form

$$
\frac{\partial}{\partial \theta} \pm x \frac{\partial}{\partial x} \quad(+ \text { if } \gamma \text { is an attractor, }- \text { if } \gamma \text { is a repeller }) .
$$

If $\gamma$ is not orientable, then $\gamma$ is in standard form if the double cover $\widetilde{V}$ of $V$ has coordinates $(\theta, x)$ with the vector field as in (3.12), i.e., $\widetilde{V}=S^{1} \times[-1,1]$ and we obtain $V$ by the relation $(\theta, x) \sim(\theta+\pi,-x)$. The vector field on $\widetilde{V}$ is compatible with the identification and induces a vector field on $V$.

An argument of Franks [3] generalizing the result of Newhouse and Peixoto [11] shows that any Morse-Smale flow is topologically conjugate to a MorseSmale flow whose periodic orbits are in standard form. Since the Conley index is topological, topologically conjugate flows have the same connection matrices, so we may assume that all periodic orbits are in standard form. We can now state our result.

Theorem 3.13. Let $\Delta$ be a connection matrix for an isolated invariant set $S$ with Morse decomposition $\left\{M_{\pi} \mid \pi \in P\right\}$ in the Morse-Smale flow $\varphi$ on a 2-manifold. Assume

(1) For each attracting periodic orbit $\gamma, \Delta(\pi, \gamma): H_{2}(\pi) \rightarrow H_{1}(\gamma)$ is nonzero for at most two $\pi \in P$.

(2) For each repelling periodic orbit $\gamma, \Delta(\gamma, \pi): H_{2}(\gamma) \rightarrow H_{1}(\pi)$ is nonzero for at most two $\pi \in P$.

(3) If $\gamma$ is attracting and orientable, choose an annular neighborhood $V$ with coordinates as in Definition 3.11. The boundary of $V$ consists of two circles, $C_{1}$ and $C_{2}$. For $i=1,2$, we have

$$
C_{i}=\bigcup_{i<\pi}\left(C_{i} \cap W^{u}(\pi)\right) \text {. }
$$

If there are two $\rho \in P$ with $\Delta(\rho, \gamma): H_{2}(\rho) \rightarrow H_{1}(\gamma)$ nonzero, then we assume that for all $\pi \in P$, if $C_{i} \cap W^{u}(\pi) \neq \varnothing$, then $W^{u}(\pi)$ does not intersect the other boundary circle. If $\gamma$ is repelling, then we assume the same thing with $W^{u}(\pi)$ replaced by $W^{s}(\pi)$, i.e., if there are two $\rho \in P$ with $\Delta(\gamma, \rho): H_{2}(\gamma) \rightarrow H_{1}(\rho)$ nonzero, then for all $\pi \in P$, the stable manifold of $M_{\pi}$ intersects at most one boundary circle. 
Given any collection $\mathscr{U}=\left\{U_{1}, U_{2}, \ldots, U_{n}\right\}$ of neighborhoods of the periodic orbits, there is a $\mathscr{U}$-refinement of $\varphi$ that has $\Delta$ as its unique connection matrix.

Assumption 3 is necessary. An example will be discussed later.

Consider a neighborhood $V$ of a periodic orbit $\gamma$ with coordinates as in Definition 3.11. Suppose $\gamma$ is attracting. The boundary of $\gamma$ consists of one circle if $\gamma$ is nonorientable, or two circles if $\gamma$ is orientable. Then

$$
\operatorname{bdy}(V)=\bigcup_{\gamma<\pi}\left(\operatorname{bdy}(V) \cap W^{u}(\pi)\right) .
$$

If $M_{\pi}$ is a saddle point, then $\operatorname{bdy}(V) \cap W^{u}(\pi)$ consists of one or two points. If $M_{\pi}$ is a repelling point or periodic orbit, then $\operatorname{bdy}(V) \cap W^{u}(\pi)$ is a 1-manifold, so it is either a collection of intervals or circles. Thus, $\operatorname{bdy}(V)$ decomposes into a collection of points and intervals (or circles). Let $C$ be a boundary circle. Then $C=J_{1} p_{12} J_{2} p_{23} \ldots J_{k} p_{k 1}$ where $J_{1}, J_{2}, \ldots, J_{k}$ are open intervals, $J_{i}=C \cap W^{u}(\pi)$ for some repeller $M_{\pi}$, and $p_{i, i+1}$ is the intersection of an orbit in the unstable manifold of a saddle with $C$. The order of the intervals and points lets us read off the connections between the repellers and saddles.

Lemma 3.14. Suppose $J_{i} \subset W^{u}(\pi), J_{i+1} \subset W^{u}(\rho)$ and $p_{i, i+1} \subset W^{u}(\tau)$ where $M_{\pi}$ and $M_{\rho}$ are repellers (either points or periodic orbits), and $M_{\tau}$ is a saddle. If $\pi \neq \rho$, then $C\left(M_{\pi}, M_{\tau}\right)$ and $C\left(M_{\rho}, M_{\tau}\right)$ each consists of a single orbit. If $\pi=\rho$, then $C\left(M_{\pi}, M_{\tau}\right)$ consists of two orbits.

Proof. Since $p_{i, i+1} \subset \operatorname{bdy}\left(J_{i}\right)$ and $p_{i, i+1} \subset \operatorname{bdy}\left(J_{i+1}\right)$, Lemma 1.13 implies that $\tau \leq \pi$ and $\tau \leq \rho$ in the flow-defined order. By transversality and the fact that $M_{\tau}$ is a saddle, there are exactly two orbits in the stable manifold of $M_{\tau}$. This establishes the assertion in the case $\pi \neq \rho$.

On the other hand, if $\tau<\sigma$ for some $\sigma \in P$, then the transversality condition implies that $C\left(M_{\sigma}, M_{\tau}\right) \neq \varnothing$. By using Hartman's theorem and working in a neighborhood of $M_{\tau}$, for either orbit in $W^{u}(\tau)$, one can construct a sequence $\left\{x_{n}\right\} \subset W^{u}(\sigma), x_{n} \rightarrow x$, and $x \in W^{u}(\tau)$. By continuity of the flow, it follows that for $n$ large, there is a $T_{n}>0$ such that $\varphi\left(x_{n}, T_{n}\right) \in J_{i}$ or $J_{i+1}$. So, if $\pi=\rho, W^{s}(\tau) \subset W^{u}(\pi)$, and so $C\left(M_{\pi}, M_{\tau}\right)=W^{s}(\tau)$.

Of course, if $\gamma$ is repelling, a similar statement holds with $J_{i}$ and $J_{i+1}$ contained in the stable manifolds of attractors and $p_{i, i+1}$ contained in the stable manifold of a saddle.

The plan of the proof of Theorem 3.13 is as follows. Suppose $\gamma$ is attracting. We replace $\gamma$ by two-doubly connected rest points $p$ and $q$, where $q$ is an attractor and $p$ is a saddle. $W^{s}(\gamma)=W^{s}(p) \cup W^{s}(q) . W^{s}(p) \cap \operatorname{bdy}(V)$ consists of two points. Transversality will be maintained if each point lies in one of the $J_{i}$, i.e., we avoid saddle-saddle connections. The connection matrix entries will be determined by the intervals in which the two points lie. Since $p$ is a saddle, the maps $H_{2}(\pi) \rightarrow H_{1}(p)$ will be flow defined, so they will count the number of connections $(\bmod 2)$. 
Suppose $\Delta(\pi, \gamma): H_{2}(\pi) \rightarrow H_{1}(\gamma)$ and $\Delta(\rho, \gamma): H_{2}(\rho) \rightarrow H_{1}(\gamma)$ are two nonzero entries (i.e. two l's) in the connection matrix row corresponding to $\gamma$. Then $\gamma<\pi$ and $\gamma<\rho, M_{\pi}$ and $M_{\rho}$ are repellers, so $J_{i} \subset W^{u}(\pi)$ and $J_{k} \subset W^{u}(\rho)$ for some $i, k$. We must show that we can replace $\gamma$ by the doubly-connected rest points in such a way that $W^{S}(p) \cap \operatorname{bdy}(V)$ has a point in $J_{i}$ and a point in $J_{k}$. If $\gamma$ is orientable, we must show that $J_{i}$ and $J_{k}$ lie in different boundary circles since the two points in $W^{s}(p) \cap \operatorname{bdy}(V)$ lie in different boundary circles. If all of the maps $H_{2}(\pi) \rightarrow H_{1}(\gamma)$ are zero, we must show that it is possible to have a double connection, i.e., if $\gamma$ is orientable, there are intervals $J_{i}$ and $J_{k}$ that lie in different boundary circles and both $J_{i}$, $J_{k} \subset W^{u}(\pi)$ for some $\pi$.

Lemma 3.15. Suppose $\gamma$ is attracting, $J_{i}$ and $J_{k}$ are intervals as indicated previously. If $\gamma$ is orientable, we assume $J_{i}$ and $J_{k}$ lie in different boundary circles. Then we can replace $\gamma$ with two doubly-connected rest points $p$ and $q$, with $p$ a saddle and $q$ an attractor (as in a $\mathscr{U}$-refinement) such that $W^{s}(p) \cap J_{i}$ and $W^{s}(p) \cap J_{k}$ are both nonempty.

Proof. Choose $V$ and coordinates $(\theta, x)$ as in Definition 3.11. Let $h(x)$ be a smooth function that is identically zero near $x= \pm 1$ and identically 1 on $[-1 / 2,1 / 2]$.

Assume $y$ is orientable. Call the boundary circle corresponding to $x=1$ the outer boundary circle and the other boundary circle the inner boundary circle. Assume $J_{i}$ is in the outer boundary circle. Replace the flow on $V$ with the flow generated by

$$
[(1-h(x))+h(x) \sin (q+a)] \frac{\partial}{\partial \theta}-x \frac{\partial}{\partial x} .
$$

There are two rest points, a saddle $p$ when $\theta+\alpha=0$, and an attractor $q$ when $\theta+\alpha=\pi$. By choosing $\alpha=\alpha_{1} \in[0,2 \pi)$ properly, we will have $W^{S}(p) \cap J_{i} \neq \varnothing$. Now replace $\alpha$ in (3.16) by a function $\alpha(x) . \alpha(x) \equiv \alpha_{0}$ if $-1 / 4 \leq x \leq 1$. By choosing $\alpha(x)$ to be an appropriate increasing function on $(-1 / 2,-1 / 4)$ we can arrange the other orbit of $W^{s}(p)$ to intersect the inner boundary circle in any point we desire. Choosing this point to be in $J_{k}$ completes the construction in the orientable case.

If $\gamma$ is nonorientable, let $\tilde{V}$ be the double cover of $V$ with coordinate $(\theta, x)$ in $\widetilde{V}$. Let $h$ be as just described, plus $h(-x)=h(x)$. This time we replace the vector field with

$$
[(1-h(x))+h(x) \sin (2 \theta+\alpha)] \frac{\partial}{\partial \theta}-x \frac{\partial}{\partial x} .
$$

In $\widetilde{V}$ there are two saddles corresponding to $p$ in $V$, and two intervals corresponding to $J_{i}$, one in each boundary circle of $\widetilde{V}$. Let $\tilde{p}$ denote the lift of $p$ with $\theta$ coordinate in $[0, \pi)$. By choosing $\alpha=\alpha_{0} \in[0, \pi)$ properly, one orbit of $W^{s}(\tilde{p})$ will intersect the boundary of $\tilde{V}$ at a point $\tilde{y}$, where $\tilde{y}$ is the lift of a point in $J_{i}$. Assume $\tilde{y}$ is in the boundary circle with $x=1$. Let 
$W$ be a small connected neighborhood of $\{\theta=0\} \cup\{$ the orbit connecting $\tilde{p}$ and $\tilde{y}\}$, which is invariant under the map $(\theta, x) \rightarrow(\theta+\pi,-x)$. By varying $\alpha=\alpha(\theta, x)$ in the component of $\widetilde{V} \backslash W$ that intersects the boundary circle $x=-1$, we can force the other orbit of $W^{s}(\tilde{p})$ to intersect the boundary circle $x=-1$ in a point $\tilde{z}$ that is a lift of a point in $J_{k}$. Finally, we define $\alpha$ in the other component of $\widetilde{V} \backslash W$ by $\alpha(\theta, x)=\alpha(\theta-\pi,-x)$. The resulting vector field is invariant under the projection $\widetilde{V} \rightarrow V$, so it gives us a vector field on $V$ with the desired properties.

A similar statement holds for $\gamma$ repelling. The next step involves proving a generalization of Theorem 1.12.

Lemma 3.17. Let $M_{\rho}$ be a saddle and $M_{\pi}$ a periodic orbit. Then $M_{\rho}$ and $M_{\pi}$ are adjacent in the flow-defined order and the boundary map $\partial$ counts the number of connecting orbits between the sets $(\bmod 2)$.

Proof. $M_{\rho}$ and $M_{\pi}$ are adjacent in the flow-defined order by transversality. Let $S=M(\pi, \rho)$, and suppose $M_{\pi}$ is attracting. Then $H(\pi)$ is $\mathbb{Z}_{2}$ in dimensions 0 and 1 , and 0 otherwise. $H(\rho)$ is $\mathbb{Z}_{2}$ in dimension 1 , and 0 otherwise. The connection matrix for $S$ with this two-set Morse decomposition is

$$
\pi_{0} \quad\left[\begin{array}{ccc}
\pi_{0} & \pi_{1} & \rho \\
0 & 0 & \partial
\end{array}\right],
$$

where $\partial$ is the flow-defined map. Now replace $M_{\pi}$ with two doubly-connected rest points, a saddle $p$ and attractor $q$ as in Lemma 3.i5. The connection matrix for the new flow is

$$
\Delta=q\left[\begin{array}{llll}
q & p & \rho \\
& 0 & 0 & \partial^{\prime}
\end{array}\right] .
$$

By Theorem 3.10, $\Delta$ is a connection matrix for the original flow. Both $\partial$ and $\partial^{\prime}$ are flow defined, hence unique, so $\partial=\partial^{\prime}$. $\partial^{\prime}$ counts the connecting orbits between $M_{\pi}$ and $q(\bmod 2)$ by Theorem 1.7. This is the same as the number of connecting orbits between $M_{\rho}$ and $M_{\pi}$, so $\partial$ counts the number of connecting orbits between $M_{\rho}$ and $M_{\pi}(\bmod 2)$.

The case for $M_{\pi}$ a repeller is similar.

Lemma 3.18. Suppose $\gamma$ is an attracting periodic orbit, $J_{i} p_{i, i+1} J_{i+1}$ is a portion of a decomposition of a boundary circle of $\gamma, J_{i} \subset W^{u}(\pi), J_{i+1} \subset W^{u}(\rho)$, and $p_{i, i+1} \subset W^{u}(\tau)$, where $\rho$ and $\pi$ are repellers and $\tau$ is a saddle. Then the pairs $\pi, \tau$ and $\rho, \tau$ are adjacent in the flow-defined order. Let $\Delta$ be a connection matrix. If $\pi \neq \rho$, then $\Delta(\pi, \tau)=\Delta(\rho, \tau)=1$, and if $\pi=\rho$, then $\Delta(\pi, \tau)=0$.

Proof. The fact that $\pi, \tau$ and $\rho, \tau$ are adjacent follows from transversality. The connection maps are flow defined and count the number of connections $(\bmod 2)$. The result now follows from Theorem 1.12 and Lemmas 3.14 and 3.17 .

Again, an analogous statement holds for $\gamma$ repelling. 
Proof of Theorem 3.13. Let $\Delta$ be a connection matrix for $\varphi$ satisfying the hypotheses of the theorem, and let $\mathscr{U}$ be given. We will show how to replace the periodic orbits with doubly-connected rest points so that the resulting flowdefined maps agree with those in $\Delta$. If $M_{\rho}$ and $M_{\pi}$ are rest points, then the map $\Delta(\rho, \pi)$ is flow defined and will not be changed by the $\mathscr{U}$-refinement. If $M_{\pi}$ is a periodic orbit and $M_{\rho}$ is a saddle, then the corresponding map in $\Delta$ is flow defined and counts the number of orbits (mod 2) between the saddle and the periodic orbit. When $M_{\pi}$ is replaced with a saddle and an attracting (or a repelling) rest point, the number of orbits between $M_{\rho}$ and the new attracting (or repelling) point will be the same as the number of connecting orbits between $M_{\rho}$ and $M_{\pi}$, so the connection matrix map will not change (as in Lemma 3.16). The only maps in $\Delta$ that the $\mathscr{U}$-refinement can change are those between repellers and attracting periodic orbits, and those between repelling periodic orbits and attractors that are not flow defined. We must show that a $\mathscr{U}$-refinement can be made so that these two collections of maps agree with those prescribed by $\Delta$.

Suppose $M_{\pi}$ is an attracting, nonorientable periodic orbit. Let $E=\{\rho \in$ $\left.P \mid \Delta(\rho, \pi): H_{2}(\rho) \rightarrow H_{1}(\pi) \neq 0\right\}$. By hypothesis, $|E|=0,1$, or 2 . We show that $|E|=0$ or 2 . Let $I=\{\rho \in P \mid \pi \leq \rho\}$. I is an interval in $P$, so $H \Delta(I) \cong H(I)$. All possible nonzero maps $\Delta(\rho, \pi)$ are contained in the restriction $\Delta(I)$. If $\rho \in I, \rho \neq \pi$, then $M_{\rho}$ is a saddle or a repeller. Let $\tau_{1}, \tau_{2}, \ldots, \tau_{n}$ index the collection $\left\{H_{1}(\rho) \mid \rho \in I, \rho \neq \pi\right\}$ and $\sigma_{1}, \sigma_{2}, \ldots, \sigma_{m}$ index the set $\left\{H_{2}(\rho) \mid \rho \in I, \rho \neq \pi\right\}$. Then $\Delta(I)$ has the following form:

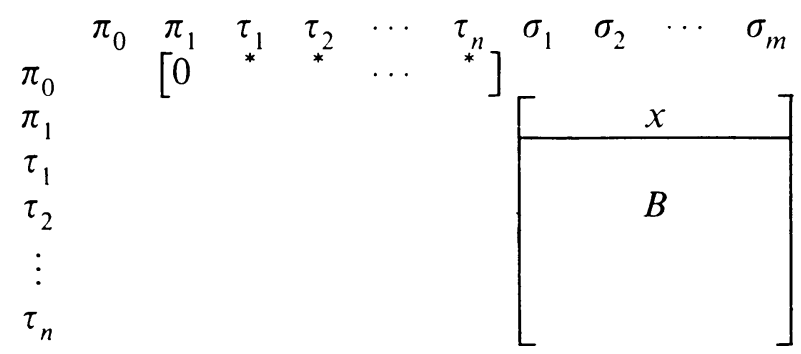

The maps in $B$ are flow defined. The maps in the second row (above $B$ ) are the maps from elements of $E$ to $M_{\pi}$. Call this row $x$. We must show that there cannot be a single 1 in $x$. Let $C$ denote the matrix whose rows are $x$ and the rows of $B . H_{2}(I)=\operatorname{ker}(C)$. Thus, the rank of $C$ is determined by the flow. We will show that $\operatorname{rank}(C)=\operatorname{rank}(B)$. To see this, let $J_{i}$ be an interval in a decomposition of the boundary of a tubular neighborhood of $M_{\pi}$. By replacing $M_{\pi}$ with two doubly-connected rest points as in Lemma 3.15, we can arrange for both orbits in the stable manifold of the new saddle $p$ to intersect $J_{i}$. In the connection matrix for the refinement, the row $x$ contains all zeroes, since $p$ is doubly connected to one repeller and not connected to any other repeller. The connection matrix for the refinement is a connection matrix for the original flow by Lemma 3.10, so $\operatorname{rank}(C)=\operatorname{rank}(B)$. 
Lemma 3.18 implies that the sum of the entries in any row of $B$ is 0 . If $x$ had a single 1 in it, then $\operatorname{rank}(C)=\operatorname{rank}(B)+1$ (since $B$ has row sum 0 ), which is impossible. So $x$ has zero or two 1's, i.e., $|E|=0$ or 2 . If $|E|=0$, replace $M_{\pi}$ with the doubly-connected saddle $p$ and attractor $q$ such that both orbits of the stable manifold of $p$ intersect the same interval $J_{i}$ in a boundary circle of a tubular neighborhood, as just illustrated. Then $x$ will contain all zeroes. If $|E|=2$, i.e. if $\Delta\left(\sigma_{i}, \pi\right)=\Delta\left(\sigma_{k}, \pi\right)=1$, choose intervals $J_{i}$ and $J_{k}$ in the boundary of a tubular neighborhood of $M_{\pi}$ with $J_{i} \subset W^{u}\left(\sigma_{i}\right)$ and $J_{k} \subset W^{u}\left(\sigma_{k}\right)$. Now when $M_{\pi}$ is replaced, we arrange for the stable manifold of the new saddle to intersect the intervals $J_{i}$ and $J_{k}$. This will give us $\Delta\left(\sigma_{i}, \pi\right)=\Delta\left(\sigma_{k}, \pi\right)=1$, so the maps in the row $x$ of the connection matrix of the refinement will agree with those in $\Delta$.

By repeating this process for each attracting, nonorientable periodic orbit $M_{\pi}$, we will have the maps in the connection matrix of the refinement agreeing with $\Delta(\rho, \pi)$ in the original connection matrix where $\Delta(\rho, \pi): H_{2}(\rho) \rightarrow$ $H_{1}(\pi)$. If $M_{\pi}$ is a nonorientable repelling periodic orbit, then an analogous construction yields a refinement where the maps $H_{1}(\pi) \rightarrow H_{0}(\rho)$ agree with $\Delta(\pi, \rho)$.

The remaining step in our construction is the replacement of the orientable periodic orbits, so let $M_{\pi}$ be an orientable attracting periodic orbit. Choose $V$ to be a small neighborhood with coordinates as in Definition 3.11. Define $E=\left\{\rho \in P \mid \Delta(\rho, \pi): H_{2}(\rho) \rightarrow H_{1}(\pi) \neq 0\right\}$. By hypothesis, $|E|=0,1$, or 2 . We must show the following:

(a) $|E| \neq 1$. When we introduce a new saddle $p$, it will either be connected to two distinct repellers (if $|E|=2$ ), or doubly connected to one repeller (if $|E|=0$ ).

(b) If $|E|=0$, then there is a $\rho$ with $W^{u}(\rho)$ intersecting both boundary circles of $V$ so that we can arrange a double connection.

(c) If $|E|=2$, i.e. $\Delta\left(\rho_{1}, \pi\right)=1$ and $\Delta\left(\rho_{2}, \pi\right)=1$, then $W^{u}\left(\rho_{1}\right)$ and $W^{u}\left(\rho_{2}\right)$ intersect opposite boundary circles of $V$.

It is clear that if (a), (b), and (c) hold, we can use Lemma 3.15 and replace $M_{\pi}$ by doubly-connected rest points so that the maps $H_{2}(\rho) \rightarrow H_{1}(\pi)$ agree with the maps in $\Delta$. As before, let $I=\{\rho \in P \mid \pi \leq \rho\}$. Again, the rank of the restriction $\Delta(I)$ is determined by the flow. All possible nonzero maps $\Delta(\rho, \pi)$ are contained in the restriction $\Delta(I)$. We use the same notation as in the nonorientable case. $\Delta(I)$ has the following form:

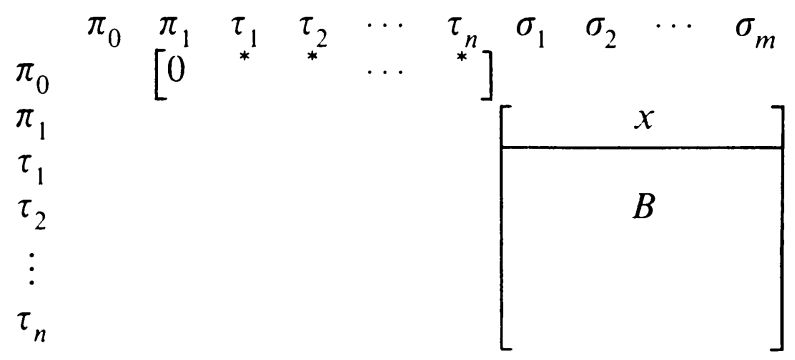


The maps in $B$ are flow defined, and the maps in the second row (above $B$ ) are the maps from the elements of $E$ to $M_{\pi}$. We distinguish two cases. Case 1 is the case where there is a $\rho \in P$ with $W^{u}(\rho)$ intersecting both boundary circles of $V$, and case 2 is the case where there is no such $\rho$.

Case 1. The same argument as the nonorientable case proves (a), since there is a $\rho$ with $W^{u}(\rho)$ intersecting both boundary circles. Thus, $|E| \neq 1$. If we are in Case 1, then $|E|=0$ by hypothesis $(3)$. Since $W^{u}(\rho)$ intersects both boundary circles for some $\rho$, Lemma 3.15 implies that we can place the new saddle so that both orbits in its stable manifold are in $V^{~ "}(\rho)$. It follows that all of the entries in the row corresponding to the saddle in the connection matrix for the refinement will be 0 , since the saddle is connected twice to $M_{\rho}$ and is not connected to any other repeller.

Case 2. Since we are assuming that for all $\rho \in P, W^{u}(\rho)$ intersects at most one boundary circle, we must show that $|E|=2$ and that the unstable manifolds of the two repellers with nonzero entries intersect different boundary circles. Let $C_{1}$ and $C_{2}$ denote the boundary circles. For $j=1,2$, define

$$
I_{j}=\left\{\rho \in P \mid \pi<\rho \text { and } W^{u}(\rho) \cap C_{j} \neq \varnothing\right\} \cup\{\pi\} .
$$

The assumption for Case 2 implies $I_{1} \cap I_{2}=\{\pi\}$ and this assumption plus Lemma 3.14 imply that $I_{1}$ and $I_{2}$ are intervals. In this case, we can further decompose $C$ into

$\left[\begin{array}{l|l}y & z \\ \hline K & 0 \\ \hline 0 & L\end{array}\right]$

where $\left[\begin{array}{ll}y & z\end{array}\right]$ is the row corresponding to the new saddle, $K$ is the block containing connection information for $I_{1}$, and $L$ contains the information for $I_{2}$.

We make an arbitrary replacement so that the new saddle is connected to two distinct repellers. In this case we have

$$
C=\left[\begin{array}{ccccccc}
0 & \cdots & 0100 & \cdots & 0010 & \cdots & 0 \\
& & K & & 0 & & \\
& & 0 & & L & &
\end{array}\right]
$$

i.e., $y$ has a single 1 and $z$ has a single 1 . Notice that the left side of $C$,

$$
\left[\begin{array}{c}
0 \cdots 010 \cdots 0 \\
K \\
0
\end{array}\right]
$$

is a connection matrix for the flow on $M\left(I_{1}\right)$ and, since each row of $K$ sums 
to zero, the rank of this matrix is $\operatorname{rank}(K)+1$. Similarly, the right half of $C$ is a connection matrix for $M\left(I_{2}\right)$ and its rank is $\operatorname{rank}(L)+1$. It follows that any connection matrix for $M\left(I_{1}\right)$ has $\operatorname{rank}=\operatorname{rank}(K)+1$. Similarly, any connection matrix for $M\left(I_{2}\right)$ has $\operatorname{rank}=\operatorname{rank}(L)+1$.

Now if $|E| \neq 2$, the row above $K$ or the row above $L$ (or both) will not have a 1 in it. This would give a connection matrix for $M\left(I_{1}\right)$ with $\operatorname{rank}=\operatorname{rank}(K)$ or a connection matrix for $M\left(I_{2}\right)$ with $\operatorname{rank}=\operatorname{rank}(L)$. This is impossible, so we must have $|E|=2$.

Finally, we need to prove (c), that if $\Delta\left(\rho_{1}, \pi\right)=1$ and $\Delta\left(\rho_{2}, \pi\right)=1$, then $W^{u}\left(\rho_{1}\right)$ and $W^{u}\left(\rho_{2}\right)$ intersect opposite boundary circles of $V$. Suppose that the conclusion of $(\mathrm{c})$ does not occur, i.e., suppose that $W^{u}\left(\rho_{1}\right)$ and $W^{u}\left(\rho_{2}\right)$ only intersect the same boundary circle, say $C_{1}$. In this case, we would have

$$
C=\left[\begin{array}{ccccc}
0 \cdots 10 \cdots 100 \cdots & & 0 \\
K & & 0 & \\
0 & & L &
\end{array}\right]
$$

However, this gives us a connection matrix for $M\left(I_{2}\right)$ that has $\operatorname{rank}=\operatorname{rank}(L)$, which is impossible. A similar contradiction occurs if $W^{u}\left(\rho_{1}\right)$ and $W^{u}\left(\rho_{2}\right)$ only intersect $C_{2}$.

By repeating this process for all attracting periodic orbits, we will have all maps $H_{2}(\rho) \rightarrow H_{1}(\pi)$ agreeing with $\Delta(\rho, \pi)$ where $M_{\pi}$ is an attracting periodic orbit. An analogous procedure for repelling orbits $M_{\pi}$ will give us the prescribed maps $H_{1}(\pi) \rightarrow H_{0}(\rho)$ where $\rho$ is an attracting fixed point (which may have come from the replacement of a periodic orbit). All other maps are flow defined, so they are unchanged by the refinement. Thus the (unique) connection matrix for the refinement with the Morse decomposition consisting of rest points will be equal to the prescribed connection matrix $\Delta$ for the original Morse decomposition.

Example 3.19. Consider the flow on the torus shown in Figure 3.20. The torus is obtained by identifying opposite sides of the rectangle. Notice that this flow does not satisfy hypothesis (3) of Theorem 3.13, since the unstable manifold of rest point 8 intersects both sides of a tubular neighborhood of the periodic orbit. The connection matrix has the form shown in the following with matrix entries $a, b, c$, and $d$ corresponding to the maps that are not flow defined. The rank condition implies that an even number of the four entries are 1 , and one possibility is $b=c=1, a=d=0$. It is possible to construct $\Phi$ 's so this choice gives us a connection matrix, but it is impossible to obtain this connection matrix by replacing the periodic orbit with a saddle and a repeller. Thus assumption (3) is necessary. Perhaps this difficulty would not arise if a field of characteristic other than two were used, but this would make the rest of the argument much more complicated. 


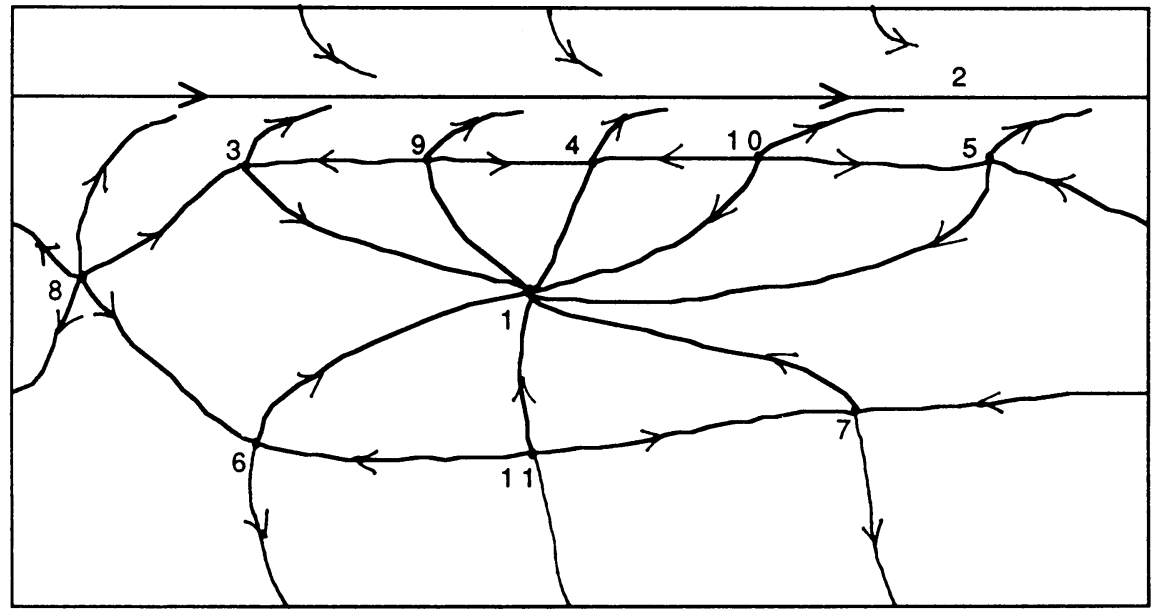

FigURE 3.20

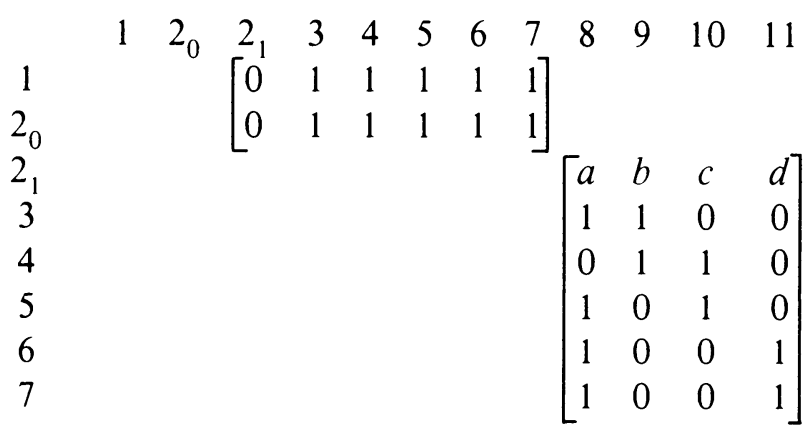

Finally, we remark that some generalization to higher dimensions is probably true. The situation is much more complicated, since two periodic orbits of the same index can be connected, and the intersection of the stable manifold of a periodic orbit with a tubular neighborhood of the orbit will have a much more complicated decomposition. We will discuss this in a future paper.

Acknowledgment. The author would like to thank the referee for helpful suggestions.

\section{REFERENCES}

1. C. C. Conley, Isolated invariant sets and the Morse index, CBMS Regional Conf. Ser. Math., no. 38, Amer. Math. Soc., Providence, R.I., 1978.

2. C. C. Conley and E. Zehnder, Morse-type index theory for flow's and periodic solutions for Hamiltonian equations, Comm. Pure Appl. Math. 37 (1984), 207-253.

3. J. Franks, Morse-Smale flows and homotopy theory, Topology 18 (1979). 199-215.

4. R. Franzosa, Index filtrations and connection matrices for partially ordered Morse decompositions, Thesis, Univ. of Wisconsin, 1984.

5. __ Index filtrations and the homology index braid for partially ordered Morse decompositions, Trans. Amer. Math. Soc. 298 (1986), 193-213. 
6. The connection matrix theory for Morse decompositions, Trans. Amer. Math. Soc. 311 (1989), 561-592.

7. _ The continuation theory for Morse decompositions and connection matrices, Trans. Amer. Math. Soc. 310 (1988), 781-803.

8. C. McCord, The connection map for attractor-repeller pairs, Trans. Amer. Math. Soc. $\mathbf{3 0 7}$ (1988), 195-203.

9. K. Mischaikow, Classification of travelling wave solutions to reaction-diffusion equations, preprint.

10. K. Mischaikow and G. Wolkowicz, Predator-prey with group defense; a connection matrix approach, preprint.

11. S. Newhouse and M. Peixoto, There is a simple arc joining any two Morse-Smale flows, Astérisque 31 (1976).

12. J. Reineck, Travelling wave solutions to a gradient systems, Trans. Amer. Math. Soc. 307 (1988).

13. D. Salamon, Connected simple systems and the Conley index of isolated invariant sets, Trans. Amer. Math. Soc. 291 (1985).

Department of Mathematics, SUNY at Buffalo, Buffalo, New York 14214 